\title{
Die deutsche IPF-Leitlinie: national vs. international
}

\author{
The German IPF Guideline: national vs. international
}

Autoren

Institute
U. Costabel ${ }^{1}$, J. Behr ${ }^{2}$

Abteilung Pneumologie/Allergologie, Ruhrlandklinik, Westdeutsches Lungenzentrum am Universitätsklinikum Essen, Essen Asklepios Kliniken München-Gauting und Medizinische Klinik und Poliklinik V, Klinikum der Ludwig-Maximilians-Universität München

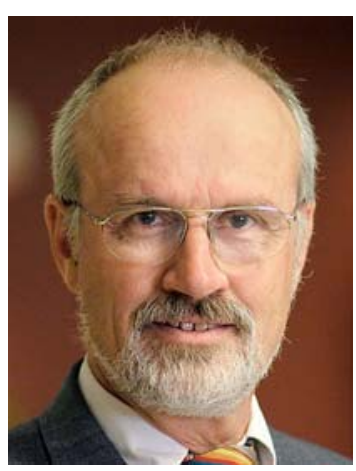

Prof. Dr. med. U. Costabel

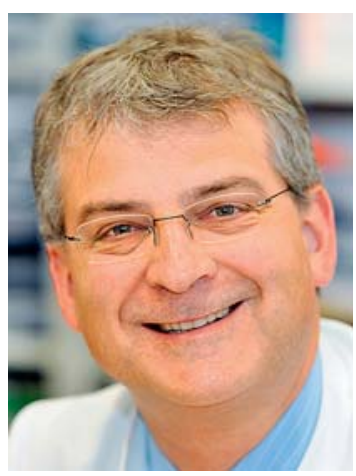

Prof. Dr. med. J. Behr

\section{Bibliografie}

DOI http://dx.doi.org/

$10.1055 / \mathrm{s}-0032-1326010$

Online-Publikation: 0.0 .

Pneumologie 2013; 67: 79-80

(c) Georg Thieme Verlag KG

Stuttgart · New York

ISSN 0934-8387

\section{Korrespondenzadresse}

Prof. Dr. U. Costabel

Ruhrlandklinik

Tüschener Weg 40

45239 Essen

ulrich.costabel@ruhrlandklinik.

uk-essen.de
Nach dem ersten Statement zur idiopathischen pulmonalen Fibrose (IPF) aus dem Jahre 2000 [1], welches als typisches Konsensus-Statement auf Expertenmeinung basierte, wurde im Jahre 2011 die internationale IPF-Leitlinie publiziert, bei welcher die Empfehlungen zur Diagnostik und Therapie nach einer evidenzbasierten GRADEMethodik anhand von konkreten Fragen verfasst wurden [2]. Die beiden Autoren dieses Editorials waren an der Erstellung der internationalen Leitlinie beteiligt. Uns und anderen europäischen Beteiligten wurde klar, dass die internationale IPF-Leitlinie inhaltlich an nationale Verhältnisse anzupassen sei. Deutsche Experten wurden ins Boot geholt und unter der Schirmherrschaft der DGP wurde am 03.12.2011 in Bochum eine Konsensuskonferenz abgehalten.

Wozu brauchen wir überhaupt eine eigene IPFLeitlinie? Warum reicht die internationale Leitlinie nicht aus? Die Beantwortung dieser Fragen ergibt sich beim Lesen der deutschen Leitlinie [3]. Die Änderungen im Vergleich zur internationalen Leitlinie sind in kursiver Schrift hervorgehoben. Neben den Besonderheiten des deutschen Gesundheitssystems und den Gepflogenheiten der deutschen Pneumologen sind auch wesentliche Änderungen in der medikamentösen Therapie aufgegriffen worden, nachdem in den letzten 2 Jahren neue, wegweisende Studien veröffentlicht wurden.

Im diagnostischen Teil der neuen internationalen IPF-Leitlinie wird herausgestellt, dass nun ein UIP-Muster im HR-CT (das bedeutet den Nachweis von subpleuralen Honigwaben) den gleichen diagnostischen Wert hat wie der Nachweis eines UIP-Musters in der chirurgischen Lungenbiopsie. Bei diesen Patienten muss zur sicheren IPF-Diagnose, im Gegensatz zum IPF-Statement aus dem Jahr 2000, keine chirurgische Lungenbiopsie mehr durchgeführt werden. Auf die Major Criteria und Minor Criteria wurde verzichtet, da diese eine Einschränkung der Diagnose der IPF auf Pa- tienten mit pathologischer Lungenfunktion bedeuten würde. Die IPF wird nun zunehmend durch Zufallsbefunde beispielsweise im Rahmen einer kardialen CT-Diagnostik erkannt, sodass die Lungenfunktion durchaus normal ausfallen kann. Nach den alten Major Criteria, die alle 4 erfüllt sein mussten, wäre die Diagnose in diesen Fällen nicht möglich gewesen. Es ist aber darauf hinzuweisen, dass die klinischen Kriterien, z.B. höheres Lebensalter, die klinischen Befunde mit Knisterrasseln und Uhrglasnägeln/Trommelschlegelfingern, der chronische Krankheitsbeginn, alle im Text des Dokumentes als Definition der Krankheit und den typischen charakteristischen klinischen Befunden, die erfüllt sein müssen, enthalten sind.

Eine Abweichung in der nationalen Leitlinie findet sich beim Stellenwert der BAL. Diese Untersuchung wird international nicht empfohlen (schwache Empfehlung dagegen), nach deutscher Expertenmeinung sollte zur Abgrenzung von Differenzialdiagnosen bei Verdacht auf IPF die BAL durchgeführt werden (schwache Empfehlung dafür). Mit der Einführung der neuen Krankheitskategorie „wahrscheinliche IPF“ in der internationalen Leitlinie wird der klinischen Realität Rechnung getragen, dass die IPF-Diagnose nicht in allen Fällen eindeutig gestellt werden kann; dabei betritt die Leitlinie Neuland, da bisher nicht geklärt ist, ob und wie sich die hierunter einzuordnenden Patienten hinsichtlich Prognose und Therapieansprechen von solchen mit definitiver IPF unterscheiden. Andererseits kann die Berücksichtigung dieser Kategorie in zukünftigen Studien wichtige neue Erkenntnisse liefern.

Bezüglich der Pharmakotherapie sind seit Publikation der internationalen Leitlinie neue Erkenntnisse hinzugekommen. In der PANTHER-Studie wurde der Triple-Therapiearm bestehend aus Prednisolon, Azathioprin und N-Acetylcystein vorzeitig abgebrochen, da deutlich mehr unerwünschte Nebenwirkungen und Todesfälle im 
Triple-Therapiearm im Vergleich zum Plazeboarm aufgetreten sind [4]. Daher wird in der deutschen Leitlinie eine stark negative Empfehlung gegen die Triple-Therapie ausgesprochen. Es bleibt bei einer schwach negativen gegen die ACC-Monotherapie. Basierend auf einer Pilotstudie [5] war die Antikoagulation in der internationalen Leitlinie mit einer schwach negativen Empfehlung versehen, was bedeutet, dass Patienten nach entsprechender Information über die Risiken damit behandelt werden können. Nach der inzwischen vorliegenden Publikation der US-amerikanischen Antikoagulationsstudie, welche wegen fehlendem Effekt und gesteigerter Mortalität in der Warfaringruppe ebenfalls vorzeitig abgebrochen wurde [6], erhielt auch diese Therapie in der deutschen Leitlinie eine stark negative Empfehlung. Da neue Daten zu Pirfenidon vorliegen $[7,8]$ und unter Würdigung der Gesamtheit der zu Pirfenidon verfügbaren Studiendaten sowie neuer Daten zur Prognoserelevanz des Studienendpunkts „Abfall der forcierten Vitalkapazität“ [9] wurde für Pirfenidon eine schwach positive Empfehlung ausgesprochen, im Gegensatz zur schwach negativen Empfehlung in der internationalen Leitlinie. Basierend auf dieser Datenlage wurde Pirfenidon zur Behandlung der IPFPatienten mit geringem oder moderatem Schweregrad in Europa zugelassen.

In diesem Editorial haben wir knapp die wichtigsten Unterschiede zwischen der nationalen und der internationalen Leitlinie zusammengefasst. Wir wünschen uns, dass die nationale Leitlinie auf nachhaltigere Resonanz als die internationale stoßen wird. Damit wird hoffentlich auch ein Beitrag geleistet, um das zukünftige Management unserer IPF-Patienten zu verbessern.

\section{Literatur}

1 American Thoracic Society. European Respiratory Society. Idiopathic pulmonary fibrosis: diagnosis and treatment: international consensus statement. Am J Respir Crit Care Med 2000; 161: 646-664

2 Raghu G, Collard HR, Egan JJ et al. An official ATS/ERS/JRS/ALAT statement: idiopathic pulmonary fibrosis: evidence-based guidelines for diagnosis and management. Am J Respir Crit Care Med 2011; 183: $788-824$

3 Behr J, Günther A, Ammenwerth $W$ et al. S2K-Leitlinie zur Diagnostik und Therapie der idiopathischen Lungenfibrose. Pneumologie 2013; 67: $81-111$

4 The Idiopathic Pulmonary Fibrosis Clinical Research Network. Prednisone, azathioprine, and $\mathrm{N}$-acetylcysteine for pulmonary fibrosis. $\mathrm{N}$ Engl J Med 2012; 366: 1968 - 1977

5 Kubo H, Nakayama K, Yanai $M$ et al. Anticoagulant therapy for idiopathic pulmonary fibrosis. Chest 2005; 128: 1475-1482

6 Noth I, Anstrom SB, de Andrade J et al. A placebo-controlled randomized trial of warfarin in idiopathic pulmonary fibrosis. Am J Respir Crit Care Med 2012; 186: 88-95

7 Noble PW, Albera C, Bradford WZ et al. Pirfenidone in patients with idiopathic pulmonary fibrosis (CAPACITY): two randomised trials. Lancet 2011; 377: 1760-1769

8 Spagnolo P, Del Giovane C, Luppi F et al. Non-steroid agents for idiopathic pulmonary fibrosis. Cochrane Database Syst Rev 2010; 9: CD003134

9 du Bois RM, Weycker D, Albera C et al. Forced vital capacity in patients with idiopathic pulmonary fibrosis: test properties and minimal clinically important difference. Am J Respir Crit Care Med 2011; 184: $1382-1389$ 